\title{
Self Repeating Surface Morphology on Ultrashort Laser Pulse-Treated Vanadium Surface
}

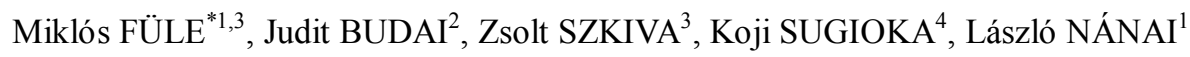 \\ ${ }^{1}$ Department of General and Environmental Physics, University of Szeged, Hungary \\ ${ }^{2}$ Department of Optics and Quantum Electronics, University of Szeged, Hungary \\ ${ }^{3}$ High Intensity Laser Laboratory, Department of Experimental Physics, University of Szeged, Hungary \\ ${ }^{4}$ Laser Technology Laboratory, RIKEN, Japan
}

\begin{abstract}
In this study the nanostructure formation on vanadium surfaces due to laser irradiation in air, water, and ethanol ambients is investigated. Two laser systems have been used for the nanostructure creation with rather different pulse lengths (Ti:sapphire laser: $22 \mathrm{fs}$ and XeCl-dye laser: $0.6 \mathrm{ps}$ ). Depending on the different ambient and different pulse durations the morphology of vanadium surface showed significant differences. In case of the fs laser pulses periodic ripple-like structures developed whose periodicity strongly depended on the applied ambient. In case of sub-ps pulses columnlike structures were observed. The nanostructure formation is discussed in terms of energy coupling between the electron phonon subsystems of the material and the properties of ambient.
\end{abstract}

DOI: $10.2961 / \mathrm{jlmn} .2014 .01 .0010$

Keywords: laser-matter interaction, surface structure, ultra short laser pulse, fractal analysis, Fourier analysis.

\section{Introduction}

Surface structure of solid state materials significantly influences several of their important properties, such as the optical behavior and chemical reactivity $[1,2]$. Therefore, along the application possibility of structured surfaces, surface modification and examination became an important part of today's materials physics. For example surfacemodified metals and metal oxides are good candidates to build different types of nanosensors or large surface chemical catalytic reactors [3-5]. Surface modification can be performed using chemical reactions with different kinds of acid and alkaline solutions. The chemical etching gives fast results, but it has some unwanted consequences, such as the toxicity of the used materials or the low controllability of these processes. To avoid these problems the laser-based methods, e.g., liquid phase pulsed laser ablation (LP-PLA) [6] seems to be a good candidate for surface modification besides the pure laser treatment in air. In this method the application of toxic materials can be avoided, and clear, controlled surface nanostructures can be produced [7-10].

Vanadium is a possible candidate for developing chemical gas sensors and chemical catalyst materials after surface oxidization $[8,11,12]$, especially in the $\mathrm{V}_{2} \mathrm{O}_{5}$ phase. Hence, a lot of studies have been devoted for its laser-based production [13-16] and preparation in thin film forms [17].

It is inevitable to achieve large surface area - volume ratios for efficient sensor and catalytic films/surfaces. In this study we have examined the surface structuring of pure vanadium surface in order to obtain samples with increased roughness and thus large surface areas. Characterization of the surfaces was performed in terms of its' fractal dimension and its' coverage by micron sized structures as both quantities are good indicator of roughness.

\section{Experiments}

In our experiments, vanadium surfaces were irradiated with two kinds of lasers in different ambients. Pulses from both laser sources were perpendicularly directed to the surface by alumina mirrors and a plane-convex lens. The mirrors reflected the beam under 45 angles of incidence. The lens was positioned just above the vanadium surface nearly at its focal length $(10 \mathrm{~cm})$ to get a narrowed beam on the surface with an almost elliptical shape. In all cases the main axes of the ellipse were 100 and $150 \mu \mathrm{m}$, respectively. The first laser source was a unique laser system. This is built up of a $\mathrm{XeCl}$ excimer oscillator and a dye laser system [18]. The wavelength, pulse energy, and pulse duration (full width at half maximum - FWHM) were: $496 \mathrm{~nm}, 150$ $\mu \mathrm{J}$ and $600 \mathrm{fs}$, respectively. This laser is referenced as a sub-ps laser in the following. The second laser is a commercially available Ti:sapphire laser oscillator, referenced later as a fs laser whose wavelength, pulse energy and pulse duration were $800 \mathrm{~nm} 260 \mu \mathrm{J}$ and $22 \mathrm{fs}$, respectively. The applied ambients were air, distilled water, and $96 \%$ ethanol. All of the used materials were laboratory purity provided from commercial partners. The vanadium surface was cleared in ultrasonic bath using ethanol before the laser treatment. It was ensured that the liquid ambients had exactly the same thickness in all cases, controlled by the volume of the liquids. The sample holder was made of polytetrafluoroethylene (PTFE) to prevent any chemical interaction. After the laser treatments the surfaces were dried in air.

The surface morphology was examined by scanning electron microscopy (SEM). A Hitachi S-4700 type electron microscope was used for surface examination. The normal operating mode was selected and the electron current and accelerating voltage were $10 \mu \mathrm{A}$ and $20 \mathrm{kV}$, respectively. For analysis of SEM images the ImageProPlus and Image 
softwares were used: for the quantitative analysis of the surface roughness the fractal dimension of the images were calculated using the box counting method (Hausdorf dimension) [19]. The coverage of the surface with nanostructures was also determined by calculating the ratio of black and white pixels, after the segmentation of the images. Finally in case of the fs laser-treated samples the SEM images were analyzed using fast Fourier transformation (FFT) to get information about the frequencies of the periodic structures on the surfaces. For this purpose 512 pixel $\times 512$ pixel sized images were cropped and Fast Fourier transformed from the original SEM images.

\section{Results}

The application of sub-ps and fs pulses results in significantly different structures. First, the outcome of the sub-ps experiments will be presented which will be followed by the results of the fs case.

\subsection{The picosecond case}

In Fig. 1. electron microscope images of the samples prepared with the sub-ps laser in different ambient with different pulse numbers is presented.

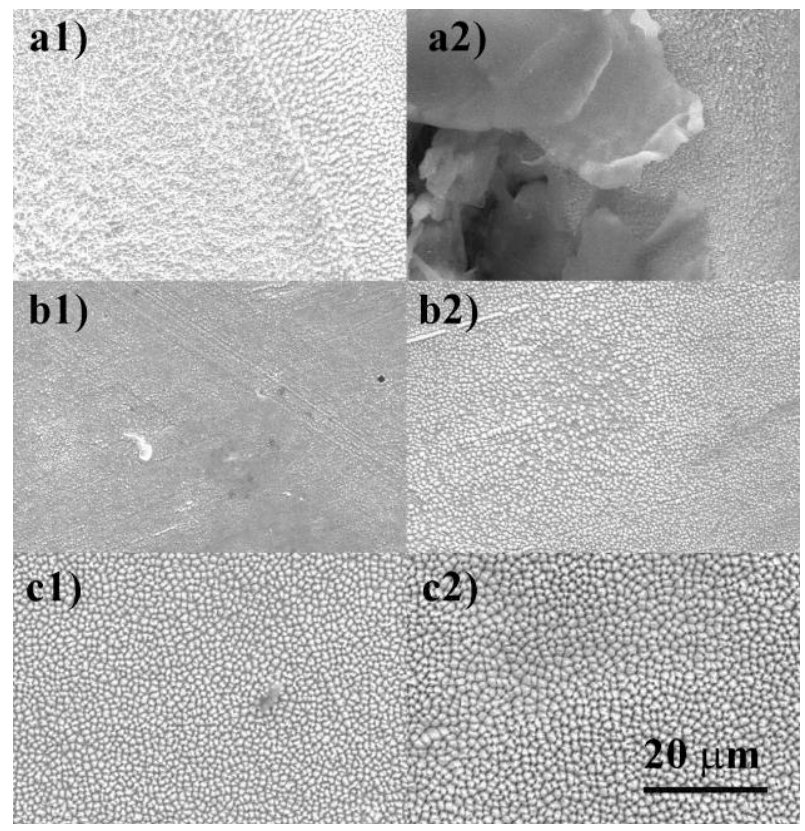

Fig. 1 electron microscope images of the samples prepared with a1), b1) and c1) 72 and a2), b2) and c2) 288 pulses of the sub-ps laser source in a) air: b) water and c) ethanol.

When the irradiation was performed in air, column-like structures appeared at the edge of the irradiated spot, while in the middle a melted texture is observed (droplet-like structures in Fig. 1. a1)), and at the highest pulse numbers even the sample was damaged (Fig. 1. a2)). Columns develop in the whole irradiated spot in case of liquid ambient. The texture emerges in case of water only at higher pulse numbers (Fig.1. b2)), while columns are most pronounced in case of the samples prepared in ethanol (Fig. 1. c1-2)). In all liquid ambients the higher pulse numbers increases the area of the columns, and clustering of the structures was observed. In order to quantitatively describe these observa- tions the fractal dimension of the images and the coverage were calculated.

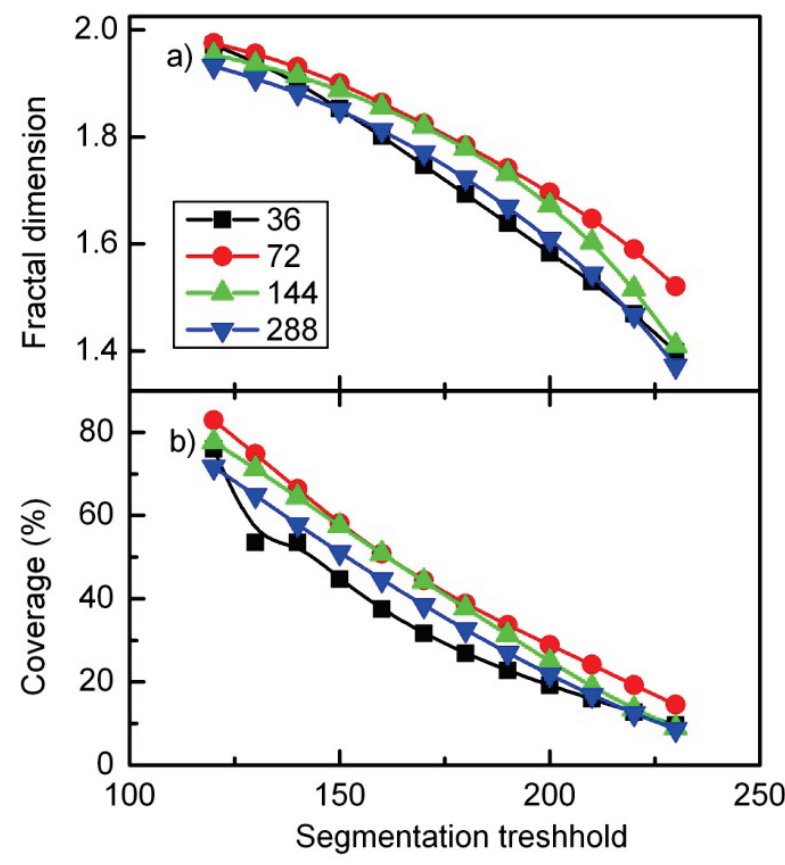

Fig. 2 The effect of segmentation threshold level on the calculated parameters on ethanol samples irradiated with different number of laser shots (indicated): a) fractal dimension, b) surface coverage.

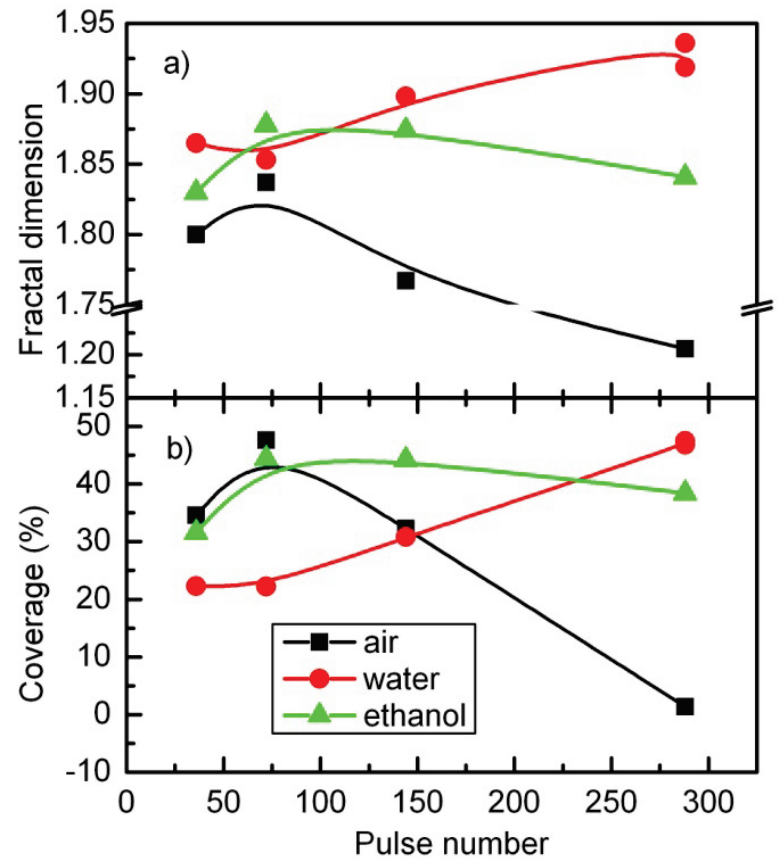

Fig. 3 a) Fractal dimension and b) surface coverage as functions of pulse number in case of the sub-ps laser treatment. Black squares, red dots, and green triangles denote samples prepared in air, water, and ethanol, respectively. Lines are presented only for guiding the eye.

Before presenting the results of these calculations we have to note that the critical parameter during the image analysis was the selection of the appropriate threshold level 
for segmentation of the image. In Fig 2.a. and b. the fractal dimension and coverage of a series of samples prepared in ethanol as a function of threshold level is presented.

In both cases the moderate threshold values (between 150 and 220 grayscale values) do not change the observed tendencies among the samples.

Therefore, later on the 170 grayscale value was chosen as a segmentation threshold, except for the images belonging to the samples treated in air with sub-ps laser pulses when the value of 220 had to be chosen.

Fig. 3 a) shows the fractal dimension of the SEM images. As it can be seen the fractal dimension increases with pulse number in case of the water-treated sample. After a steep increase at lower pulse numbers, the fractal dimension of samples prepared in ethanol slightly decreases. In case of the samples prepared in air, the highest pulse numbers causes direct ablation in the middle of the laser spot (c.f. Fig. 1 a2)). This is unfortunately followed by the evaporation of the column-like structures, therefore a decrease in the fractal dimension vs. pulse number plot is observed, showing that roughness decreases. (For the analysis of the air-treated samples the destruction in the middle of the spot was excluded from the image.)

Fig. 3 b) shows the coverage of the surface with the column like structures. As it can be seen the fractal dimension data and coverage data behave similarly when following the changes within the certain sample series: coverage increases with pulse number in case of water-treated samples, and after an increase at lower pulse numbers, the coverage of samples prepared in ethanol decrease slightly. As column-like structures are destroyed when the highest pulse numbers are applied in air, the coverage decreases.
The electron microscope images of the samples prepared with the fs laser are shown in Fig. 4. At the lowest pulse numbers globular particles appear. As pulse number is increased a ripple-like structure develops. The developing stripes act as background for initiative of pillars emerging at higher pulse numbers. The background ambient plays role not only in the size and density of these pillars, but also influences the width of the stripes. In case of the spots treated in air the stripes seem to be equidistant in the middle, and they are rarely covered with columns (Fig. 4. a)). However, we have to note, that at the perimeter of the spot the stripes are densely covered with columns. The columns are most pronounced in case of the spots treated in water (Fig. 4. b3) b4)), and the widest stripes can be observed also on these samples. However, the observed stripes emerge with different width, i.e. the structure does not seem to be periodic. Samples prepared in ethanol (Fig. 4.c)) show low numbers of columns, while they are characterized by the finest stripe system.

To characterize the observed ripple like structure $512 \times$ 512 pixel sized parts have been cropped and Fourier transformed from the SEM images. The transformed images, presented in Fig. 5, support the observations that the periodic ripples develop at around 36 and 144 pulse number in case of the samples treated in air and ethanol, respectively, as distinct maxima appear first at these pulse numbers. The Fourier transformed images of the two series differ, as the maxima are less sharp and are placed more rarely in case of the ethanol treated spots. In accordance with the observation that stripes with different width appear in case of the samples prepared in water, the Fourier transform does not exhibit a well defined maxima, but instead a ring like structure appears on the transformed image. This is the consequence of a less well defined, rather amorphous structure developing on these samples.

\subsection{The femtosecond case}

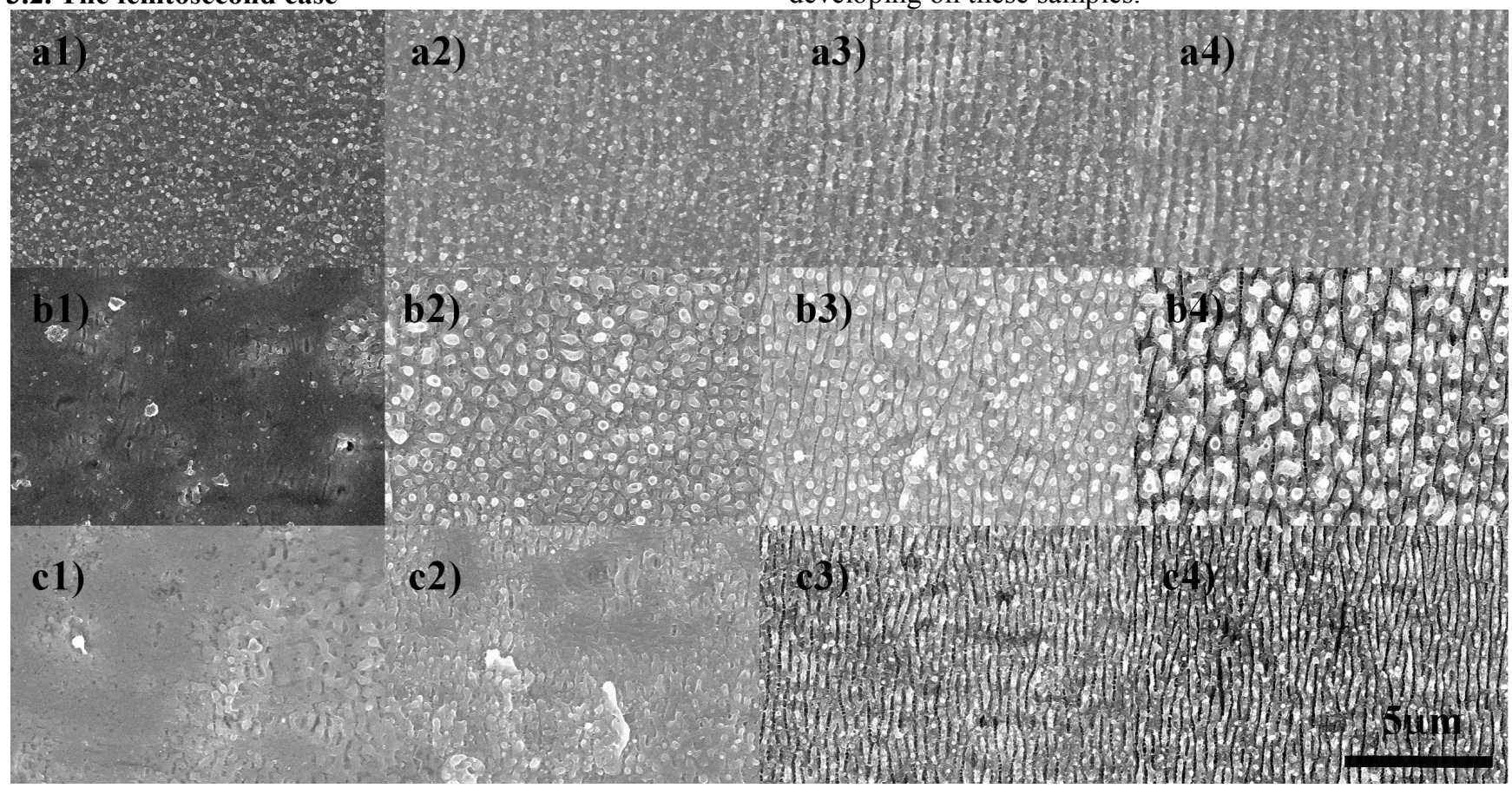

Figure 4. Scanning electron microscope images of the samples irradiated with 1) 10,2) 36, 3) 144 and 4) 360 pulses of the fs laser source in case of treatment in a1-a4 air, b1-b4 water and c1-c4 ethanol. 


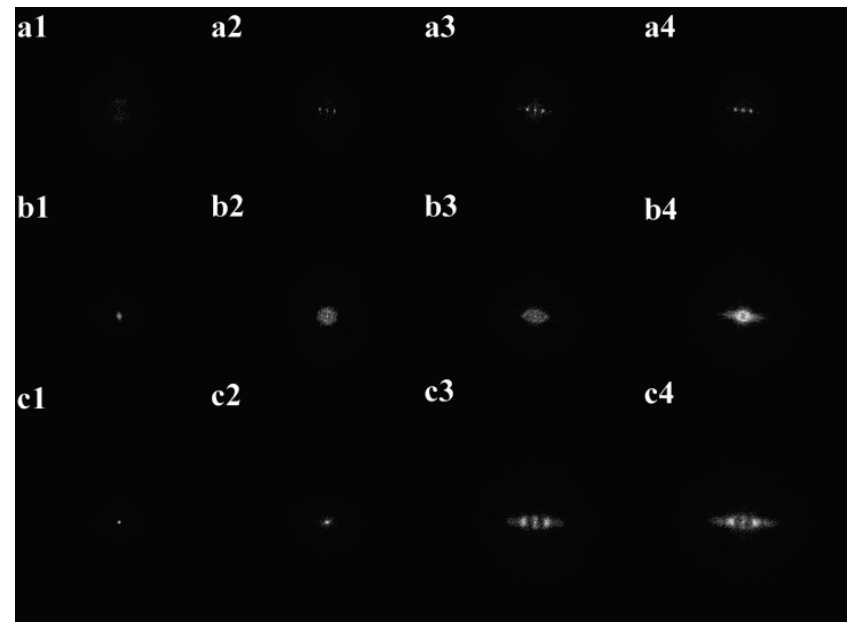

Figure 5. 2D Fourier transformed SEM images of the samples irradiated with 1) 10, 2) 36, 3) 144 and 4) 360 pulses of the fs laser source in case of treatment in a1a4 air, b1-b4 water and c1-c4 water.

To qualitatively characterize the ripples, their width have been determined from the Fourier transformed images by determing the spatial frequency belonging to the maxima in case of the samples treated in air and ethanol.

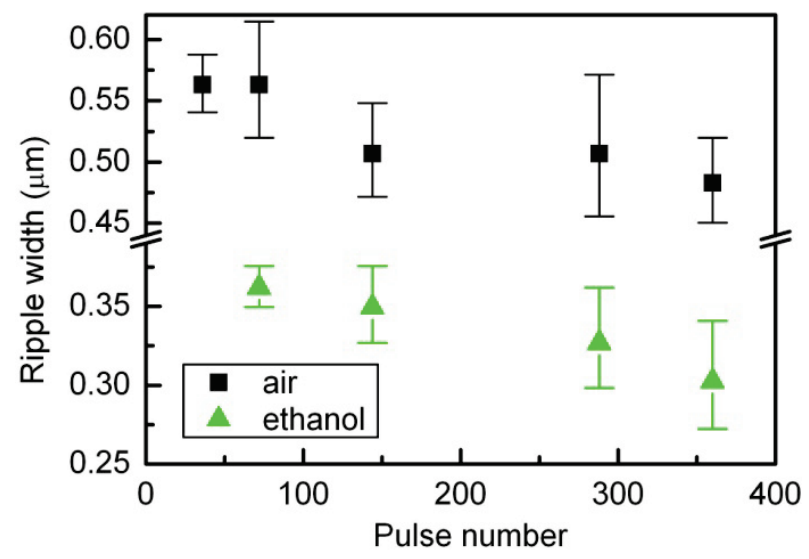

Figure 6. Ripple width as function of pulse number in case of samples treated in air (black squares) and ethanol (green triangles) using fs laser pulses. Error bars denote full width at half maxima values of the amplitude maxima in the 2D FFT images.

In case of ethanol treated sample data is not presented for 36 pulse number, as periodic structure was not observed on this sample. The ripple width values are plotted in Fig.6, where error bars present the full width at half maximum (FWHM) values. As can be seen ripples with smaller width develop in case of the air treatment. Furthermore, the ripple width decreases in both ambients with pulse number, which is followed by the increase of FWHM values.

Fractal dimension and coverage of the samples irradiated with fs laser pulses are plotted in Fig. 7 a) and b), respectively. Tendencies observed for the two data are similar: in case of air and water treated samples the two data increase at low pulse numbers and show saturation at higher pulse numbers. In case of ethanol treated samples the first pulse numbers decrease slightly the fractal dimension and cover

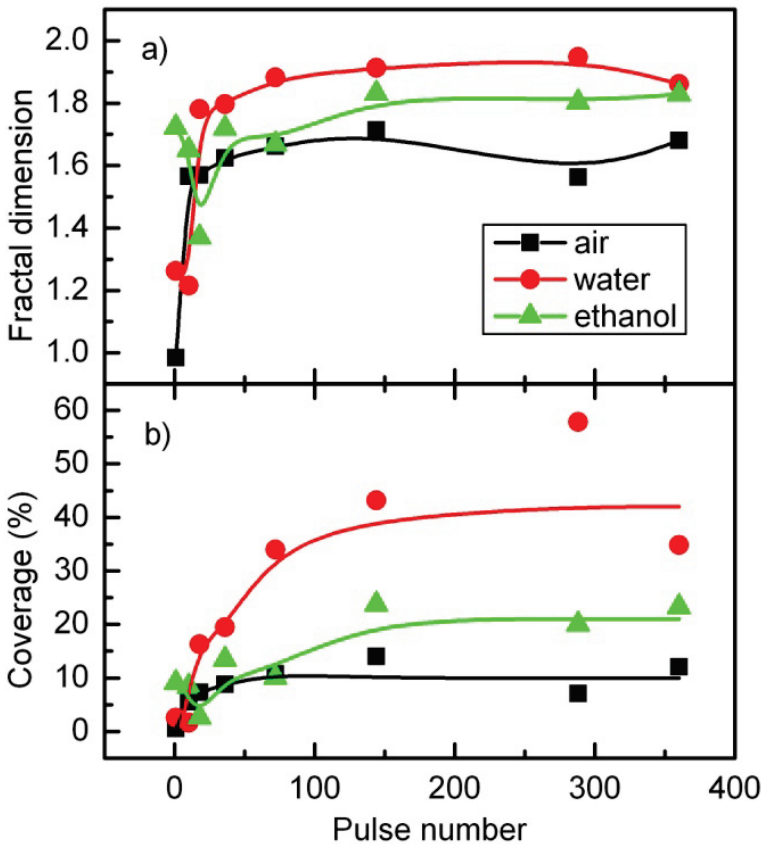

Fig. 7. a) fractal dimension and b) surface coverage as function of pulse number. Black squares, red dots and green triangles denote samples prepared in air, water and ethanol, respectively. Lines are only presented for guiding the eye.

age, but at higher pulse numbers the values increase and saturate.

\section{Discussion}

In case of irradiation of metal surfaces with laser pulses the volume of interaction can be characterized via the penetration depth of light. Penetration depth of a given material strongly depends on the wavelength of irradiating light. In the present study besides pulse length the central wavelength and pulse energy was different for the two lasers. Due to the different wavelengths the penetration depth of the ps laser is 1.64 times as large as that of the fs laser (20 $\mathrm{nm}, 12 \mathrm{~nm}$ [20]) and due to the different pulse energies the fluence of the ps laser is 0.577 smaller than that of the fs laser (22 fs: $2.2 \cdot 10^{14} \mathrm{~J} / \mathrm{cm}^{2}, 600 \mathrm{fs}: 1.27 \cdot 10^{12} \mathrm{~J} / \mathrm{cm}^{2}$ ). Thus penetration depth and fluence values of the two experiments were different, but were in the same order. However the two sources differed significantly in their intensities (22 fs: $1 \cdot 10^{14} \mathrm{~W} / \mathrm{cm}^{2}, 600 \mathrm{fs}: 2.12 \cdot 10^{12} \mathrm{~W} / \mathrm{cm}^{2}$ ) due to the considerable difference in pulse length. These together suggest that the significant differences observed in the results of the two experimental series are mainly due to the difference in the pulse length of the two applied lasers.

In case of the sub-ps pulses, the pulse length (FWHM $600 \mathrm{fs}$ ) is larger than the onset time of the electron-phonon scattering $[21,22]$, therefore the electron-phonon coupling, i.e. energy transfer to the lattice might start. According to our observations this results in the formation of micrometer-sized pillar-like structures at this laser fluence $(1.272$ $\mathrm{J} / \mathrm{cm}^{2}$ ). In case of irradiation in water and ethanol the coverage of the surface with the columns and the fractal di- 
mension of the SEM images showed increase and saturation. Unfortunately in case of dry irradiation high pulse numbers result in significant surface damages and particle evaporation reflected by steep decrease in fractal dimension and coverage. The observation that surface damage does not occur in the liquid ambient at same fluence than air ambient might be attributed to the presence of uncompressible ambient, which does not allow the free spreading of the plasma plume [23].

In case of the fs pulses, the pulse length ( $22 \mathrm{fs})$ is shorter than the time needed for energy transfer between the electron subsystem and lattice to take place within the pulse length. Therefore energy is not transferred to the lattice, ablation occurs in the oxide layer covering vanadium due to Coulomb explosion and solid-vapor transition [24] in the metal according to intensity distribution determined by interference [25]. Interference appears between the incoming wave and the wave scattered by surface structures [25], appearing in the form of globular particles (Fig. 4 a1) b1)) and $\mathrm{c} 1)$ ) due to the first $10-36$ pulses.

Based on the observation that nanostructured columns could be observed rather in case of longer pulses, when intensities are smaller and electron-phonon interaction might just start $[21,22]$, we can conclude that formation of these pillars in case of vanadium is mainly driven by the just starting thermally based processes, also shown on single crystal alloy CMSX-4 [24].

\section{Conclusion}

In this work the morphology of vanadium surfaces treated with fs and sub-ps lasers was analyzed. We found that laser irradiation with rather different pulse lengths results in fundamentally different surface structures. When the pulse length (600 fs) was enough for the electron-phonon coupling to start micrometer-sized column-like structure were observed, while in case of shorter laser pulses (22 fs) the ripple-like structure appear with only the initiative of the columns on their top. Furthermore we have shown that the coverage of the surface with the columns and the periodicity of the ripples are influenced by the chemical composition of the ambient.

\section{Acknowledgments}

Discussions with Dr. Zsolt Tóth concerning periodic surface structures are highly acknowledged. This work was supported by University of Szeged, Gyula Juhasz Teacher Training Faculty under contact number: 2S753 1601, 2S511 1601. The project was partially funded by „TÁMOP-4.2.2.A-11/1/KONV-2012-0060 - „Impulse lasers for use in materials science and biophotonics" is supported by the European Union and co-financed by the European Social Fund.

\section{References}

[1] R. M. Öksüzoğlu, P. Bilgiç, M. Yıldırım, O. Deniz, Influence of post-annealing on electrical, structural and optical properties of vanadium oxide thin films, Optics \& Laser Technology, 48, 102-109 (2013)

[2] W. Reichl, K. Hayek, The vanadium subsurface alloy on polycrystalline rhodium: formation and catalytic properties, Journal of Catalysis, 222, 53-64 (2004)
[3] E. Comini, C. Baratto, G. Faglia, M. Ferroni, A. Vomiero, G. Sberveglieri, Quasi-one dimensional metal oxide semiconductors: Preparation, characterization and application as chemical sensors, Progress in Material Science, 54, 1-67 (2009)

[4] A. Kolmakov, M. Moskovits, Chemical Sensing and Catalysis by One Dimensional Metal-oxide Nanostructures, Annual Review of Materials Research, 34, 151180 (2004)

[5] Po-Chiang Chen, GuoZhen Shen, , Chongwu Zhou, Chemical Sensors and Electronic Noses Based on 1-D Metal Oxide Nanostructures,Nanotechnology, 7, 668682 (2008)

[6] Zijie Yan, Douglas B. Chrisey, Pulsed laser ablation in liquid in for micro-/nanostructure generation, Journal of Photochemistry and Photobiology C: Photochemistry Reviews, 13, (2012) 204-223

[7] Takeshi Sasaki, Yoshiki Shimizu, Naoto Koshizaki, Preparation of metal oxide-based nanomaterials using nanosecond pulsed laser ablation in liquids, Journal of Photochemistry and Photobiology A: Chemistry, 182, (2006), 335-341

[8] S. Beke, A review of the growth of V2O5 films from 1885 to 2010, Thin Solid Films, 519, (2011) 17611771

[9] Yin Yang, Min Shi, Qian-Fei Zhou, Yue-Sheng Li, Zheng-Wen Fu, Platinum nanoparticle-graphene hybrids synthesized by liquid phase pulsed laser ablation as cathode catalysts for Li-air batteries, Electrochemistry Communications, 20(2012) 11-14

[10]Dongsik Kim, Deoksuk Jang, Synthesis of nanoparticles and suspensions by pulsed laser ablation of microparticles in liquid, Applied Surface Science, 253 (2007) 8045-8049

[11]Chien-Tsung Wang, Miao-Ting Chen, Vanadiumpromoted tin oxide semiconductor carbon monoxide gas sensors, Sensors and Actuators B: Chemical, 150, (2010), 360-366.

[12] Wei Jiang, Bin Liang, Fabrication of structured vanadium catalyst for $\mathrm{SO}_{2}$ conversion, Applied Catalysis A: General, 311 (2006), 1-7

[13]S. Beke, L. Kőrösi, S. Papp, A. Oszkó, L. Nánai, XRD and XPS analysis of laser treated vanadium oxide thin films, Applied Surface Science 255, 9779-9782, (2009)

[14]S. Beke, S. Giorgio, L. Kőrösi, L. Nánai, W. Marine, Structural and optical properties of pulsed laser deposited V2O5 thin films, Thin Solid Films 516, 46594664, (2008)

[15] S. Beke, L. Kőrösi, S. Papp, L. Nánai, J.G.Kiss, V. Safarov, Nd:YAG laser synthesis of nanostructural V2O5 from vanadium oxide sols: Morphological and structural characterizations, Applied Surface Science 254, 1363-1368 (2007)

[16] S. Beke, L. Körösi, L. Nánai, F. Brandi, In-situ optical emission spectroscopy of laser-induced vanadium oxide plasma in vacuum (invited, Rapid Communication) Vacuum, 86, 2002-2004, (2012)

[17]R. Irani, S.M. Rozati, S. Beke, Structural and optical properties of nanostructural V2O5 thin films deposited by spray pyrolysis technique: Effect of the substrate 
temperature, Materials Chemistry and Physics 139, 489-493, (2013)

[18]S. Szatmari, High-Brightness Ultraviolet Excimer Lasers, Applied Physics B, 58, 211-223 (1994)

[19] G. Bhanot, D. Duke, R. Salvador, Fractals and interpolating dimensions, Physics Letters B, 165 (1985) 355360

[20] Handbook of Optical Constants of Solids, Edited by E. D. Palik, 1997, ISBN: 978-0-12-544415-6

[21] H. Petek, S. Ogawa, Progress in Surface Science, 56 (1997) 239-310

[22] László Nánai, Szabolcs Beke, Koji Sugioka, Ultrafast Time-Resolved Spectroscopy (invited book chapter) in Femtosecond-Scale Optics (2011) Publ.: Intech

[23]Rok Petkovšek and Peter Gregorčič, Journal of Applied Physics 1020449092007

[24]N. M. Bulgakova, R. Stoian, A. Rosenfeld, I.V. Hertel, W. Marine, E.E.B. Campbell, Appl. Phys. A 81, 345356 (2005)

[25] Wei Zhang, Guanghua Cheng, Qiang Feng, Laimei Cao, Picosecond laser induced formation of spikes in a single crystal superalloy, Applied Surface Science, 258 (2012) 9452-9456

(Received: August 19, 2013, Accepted: January 15, 2014) 